\title{
LES HÔTES DES ACANTHOCÉPHALES. II - LES HôTES DÉFINITIFS. 1. POISSONS
}

\author{
Y. J. GOLVAN*, I. DE BURON**
}

RÉSUMÉ. A partir des publications qui nous étaient accessibles, nous avons tenté d'établir la liste des espèces de Poissons hébergeant des Acanthocéphales adultes, c'est-à-dire des femelles contenant des œufs mârs. C'est ce seul critère qui permet d'affirmer que ce poisson est bien l'hôte définitif vrai du parasite. Ce travail très préliminaire montre que la spécificité des Acanthocéphales pour leur hôte définitif est plus étroite qu'on ne l'a dit. S'il est exact que les Acanthocéphales, consiclérés comme "primitifs ", sont parasites de Poissons également "primitifs ", dans l'immense majorité des cas, il semble bien que ce soit le comportement trophique du poisson qui soit le facteur majeur déterminant l'infestation.

Mots-clés : Acanthocéphales. Poissons. Spécificité. Hôte définitif.

\section{Hosts of Acanthocephala. II - Definitive hosts. 1. Fishes.}

SUMMARY. From an attentive study of the largest number of available publications, we tried to establish the list of fish species harbouring fully mature Acanthocephala, i. e. females with completely developed eggs. This character is the sole on which we are allowed to assure that this fish is I ruely a definitive host for the parasite. This very preliminary work shows that acanthocephalan specificity for the definitive host is narrower than it was generally said. If few so called "primitive " Acanthocephala are indeed parasitic in "primitive "fishes, in the majority of cases the main factor for infestation is related to the trophic behaviour of the definitive host and do not depends on its phylogenic position.

Key-words : Acanthocephala. Fish. Specificity. Definitive host.

\section{Introduction}

En 1957, l'un de nous écrivait : On peut conclure en affirmant que nos connaissances actuelles sur la biologie des Acanthocéphales sont trop fragmentaires, trop imprécises, pour qu'il soit permis de déclarer, comme on le fait trop souvent, que ces Helminthes ne présentent aucune spécificité pour les hôtes qu'ils parasitent successivement au cours de leur cycle évolutif.

Aujourd'hui la liste des hôtes définitifs des Acanthocéphales s'est beaucoup allongée mais, pour une trop large part, elle n'apporte pas de renseignements de valeur sur ce problème de la spécificité pour les hôtes définitifs. Depuis près de 200 ans on a signalé tous les hôtes dans l'intestin desquels on a trouvé des formes qualifiées d'adultes mais il semble bien que beaucoup de ces hòtes "définitifs" ne soient, en réalité, que des hôtes paraténiques ou accidentels. Dans ce cas, ils

* Laboratoire de Parasilologie, Faculté de Médecine de Paris-Saint-Anloine, Cniversité Pierreet-Marie Curie, 27, rue Chaligny, F 75571 Paris Cedex 12.

** Laboratoire de Parasitologie comparée, Universilé des Sciences el Techniques du Languedoc, Faculté des Sciences, place Eugène-Bataillon, F 34060 Montpellier Cedex.

Accepté le 29 février 1988. 
ne permettent souvent pas aux femelles de mûrir parfaitement leurs œufs et ils ne jouent donc aucun rôle dans la réalisation du cycle de développement du parasite.

Toutefois, dans les dernières décennies, des travaux remarquables nous ont appris que, chez certaines espèces d'Acanthocéphales, existait une étroite spécificité pour les hôtes définitifs. Nous avons entrepris de rechercher dans la littérature tous les hôtes définitifs " vrais " des Acanthocéphales et d'en dresser la liste.

\section{Méthodes}

Pour ce travail nous n'avons retenu qu'un critère absolu : la présence signalée par les auteurs d'oufs mûrs dans le corps de la femelle adulte d'Acanthocéphale. Lorsque la présence d'œufs n'est pas signalée dans le texte ou sur les figures se rapportant à un hôte précis, nous avons délibérément écarté cet hôte de notre liste. Tous les autres critères ont été considérés comme insuffisants, entre autres la présence de l'Acanthocéphale dans l'intestin de l'hôte, la taille et le développement des organes génitaux, la fragmentation des masses ovariennes. Ce long travail de compilation est difficile à réaliser et nous avons certainement fait de nombreuses erreurs ou omissions.

Les omissions s'expliquent surtout par le fait que certaines publications nous ont été inaccessibles.

Les erreurs ont plusieurs causes :

1 - elles peuvent être de notre seule faute et nous nous en excusons auprès des auteurs qui en ont été les victimes;

2 - elles peuvent être liées à la détermination de l'hôte. Dans certains cas, l'erreur est manifeste et peut avoir des causes multiples dont la plus fréquente est l'erreur d'étiquetage. Par exemple, tel Acanthocéphale dont toutes les espèces congénériques sont parasites de poissons de mer sera signalée chez un oiseau granivore continental;

3 - elles peuvent être liées à la détermination de l'Acanthocéphale. La seule morphologie s'avère parfois insuffisante pour séparer les espèces et certains caractères considérés comme fiables ont, en réalité, une valeur très incertaine, y compris le nombre de crochets sur le proboscis et le nombre de crochets par file. Il existe de longues listes d'hôtes "définitifs " dans lesquelles tous les "Echinorhynchus" trouvés chez des poissons marins sont invariablement nommés Echinorhynchus gadi. Nous avons peut-être tort mais nous avons considéré cette détermination comme douteuse et nous n'en avons pas tenu compte dans ce travail. Dans la mesure du possible, nous avons tenu à donner les noms d'auteurs des hôtes et ceux des Acanthocéphales, ce qui réduit la marge d'erreurs;

4 --- les noms des hôtes et des Acanthocéphales sont corrects mais l'on ne peut savoir si les femelles adultes du parasite contiennent ou non des oeufs parfaitement développés. Ce cas est très fréquent. Pratiquement toutes les publications qui portent sur le contrôle (survey) du parasitisme d'une faune locale de Vertébrés ont été, pour nous, inutilisables car elles ne donnent aucune indication sur la localisation du parasite chez l'hôte et surtout n'indiquent pas si les femelles contiennent des ceufs mûrs. 


\section{Résultats}

Ils sont rapportés dans la liste qui suit et pour la lecture de laquelle nous devons fournir quelques explications complémentaires. Lorsque le nom de l'Acanthocéphale est suivi d'un chiffre entre parenthèses en italiques gras, ce chiffre renvoie à la référence qui porte ce numéro dans notre bibliographie. Nous n'avons cité ici que les publications qui sont différentes de la publication de description originale ou de celle qui a introduit une modification dans la nomenclature.

Par exemple, si le nom Telosentis molini (O. von Linstow, 1901) Van Cleave, 1923 n'est suivi d'aucun chiffre entre parenthèses, cela signifie que la présence d'œufs mûrs a été signalée soit par von Linstow, soit par Van Cleave, soit par ces deux auteurs et ni l'une ni l'autre de ces références ne figure dans notre bibliographie, ceci afin de ne pas la "gonfler " démesurément.

En revanche, si Telosentis molini (O. v. L.) V. C. est suivi de l'indication (44), cela signifie que la présence de femelles ovigères a été signalée expressément dans la publication qui porte le numéro 44 dans notre bibliographie (dans l'exemple choisi, il s'agit de Kostylew, 1926).

Pour que ceux de nos lecteurs qui ne sont pas familiers de la nomenclature et de la phylogénie des Acanthocéphales, nous avons cru bon d'ajouter d'autres indications. L'ordre auquel l'espèce appartient est indiqué par la lettre $P$ pour Palaeacanthocephala ou $\mathrm{E}$ pour Eoacanthocephala. La famille est indiquée par les 2 chiffres qui suivent et les Familles sont rangées selon la classification phylogénique que nous avons déjà proposée et qui est la suivante :

Palaeacanthocephala
01 - Illiosentidae
06 - Heteracanthocephalidae
02 - Leptorhynchoididae
07 -- Arhythmacanchidae
03 - Rhadinorhynchidae
08 -..- Hypoechinorhynchidae
04 - Fessisentidae
09 - Echinorhynchidae
05 - Diplosentidae
10 ... Pomphorhynchidae

Eoacanthocephala

01 -- Tenuisentidae 02 - Quadrigyridae 03 - Neoechinorhynchidae

Par exemple, Leplorhynchoides plagicephalus (W.) K. P-02 signifie que cette espèce qui est un Palaeacanthocephale appartient à la Famille des Leptorhynchoididae qui est, selon nos conceptions phylogéniques, l'une des plus primitives de l'Ordre.

Quant à la Famille des Polyacanthorhynchidae, elle est conservée incerlae sedis (IS) dans notre liste car nous considérons qu'elle présente des caractères qui ne permettent pas de la classer dans l'un ou l'autre de ces deux Ordres d'Acanthocéphales parasites de Poissons.

Nous sommes conscients que cette liste est beaucoup trop restrictive mais il nous a semblé nécessaire de pécher plus par défaut que par excès. Nos successeurs combleront nos lacunes et corrigeront nos erreurs. 


\title{
CLASSE DES POISSONS (PISCES)
}

SOUS-CLASSE DES Osteichthyes

\author{
CHONDROSTEI \\ ORDRE deS ACIPENSERIFORMES \\ Famille des ACIPENSERIDAE
}

Acipenser guldenstaedti Brandt Acipenser ruthenus

Acipenser stellatus Pallas, 1771

Amia calva Linne, 1758

Alosa alosa (Linné, 1758) (= Hikai.)

Dorosoma cepedianum (LeSueur)

Nematalosa nasus (Bloch)

Coilia ectenes Jordan et Seale

Coilia mystus (Linné,1758)

Engraulis encrasicolus (Linné,1758)

Lycengraulis sp.

Setipinna phasa (Hamitton-Buchanan)
Harengula ilishe (Hamihon-Buchanan)

Leptorhynchoides plagicephalus (W.) K. (27)

Leptomynchoides plagicephalus (W.) K.

Leptorhynchoides plagicephalus (W.) K.

P-02

P-02

P-02

\section{HOLOSTEI \\ ORDRE des AMIIFORMES \\ Famille des AMIIDAE}

$\begin{array}{ll}\begin{array}{l}\text { Acanthocephalus dirus (VC.) VC. et T. (2) } \\ \text { Pomphorhynchus kcyi W.et R. }\end{array} & \text { P-09 } \\ \begin{array}{l}\text { TELEOSTEI } \\ \text { P.10 }\end{array} & \\ \text { ORDRE des CLUPEIFORMES } & \\ \text { SOUS-ORDRE des CLUPEOIDEI } \\ \text { Famille des CLUPEIDAE }\end{array}$

Famille des DOROSOMIDAE

Gracilisentis gracitisentis (VC.) VC. (36)-(40) E-03

Tanaorhamphus longirostris (VC.) H.B.W. E-03

Neoechinorhynchus nematalosi T. E-03

Famille des ENGRAULIDAE

Acanthogyrus (Acanthosentis) similis (W.)A. E-02

Neoechinoihynchus coillae Y. E-03

Telosentis exiguus (VL.) VC. (44) P-01

Neoechinorhynchus macronucleatus MF. E-03

Acanthogyrus (Acanthosentis) indicus (T.) C. E-02

\section{SOUS-ORDRE des ELOPOIDEI}

Famille des ALBULIDAE

Albula vulpes (Linné, 1758)
Illiosentis africanus G. (55)

Tegorhynchus furcatus (VC. et L.) B.et M. (54)
P-01

P-01 


\section{SOUS-ORDRE deS SALMONOIDEI Famille des SALMONIDAE}

Cristivomer namaycush (Walbaum)

Oncorhynchus kisutch (Walbaum)

Oncorhynchus nerka (Walbaum)

Oncorhynchus tshawytscha (Walbaum) Salmogairdneri Richardson

Salmo gairdneri irideus Gibbons Salmo ischchan Kessler

Salmo salar Linné, 1758

Salmo trutta Linné,1758

Salvelinus fontinalis (Mitchill)

Salvelinus malma (Walbaum)

Salvelinus oquassa marstoni

Osmerus mordax (Mitchill)

Coregonus albulus

Coregonus artedii LeSueur

(= Leucichthys a.)

Coregonus clupaeaformis (Mitchill)

Coregonus hoyi

Coregonus lavaretus L.

Prosopium transmontanus

Prosopium williamsoni (Girard)

Thymallus arcticus (Pallas)

Thymallus thymallus (Linné)

$\begin{array}{ll}\text { Acanthocephalus dirus (VC.) VC. et T. (3) } & \text { P-09 } \\ \text { Echinorhynchus leidyi VC. } & \text { P-09 } \\ \text { Echinorhynchus coregoni (L.) in VC. } & \text { P-09 } \\ \text { Acanthocephalus dirus (VC.) VC. et T. (3) } & \text { P-09 } \\ \text { Acanthocephalus echigoensis F. } & \text { P-09 } \\ \text { Neoechinorhynchus rutili (O.F.M.) H. } & \text { E-03 } \\ \text { Neoechinorhynchus salmonis L.-C. } & \text { E-03 } \\ \text { Acanthocephalus dirus (VC.) VC. et T. (3) } & \text { P-09 } \\ \text { Acanthocephalus dirus (VC.) VC. et T. (3) } & \text { P-09 } \\ \text { Echinorhynchus lateralis L. (72) } & \text { P-09 } \\ \text { Neoechinomynchus salmonis L.-C. } & \text { E-03 } \\ \text { Neoechinorhynchus rutili (O.F.M.) H. } & \text { E-03 } \\ \text { Acanthocephalus dirus (VC.) VC. et T. (3) } & \text { P-09 } \\ \text { Echinorhynchus sevani D. } & \text { P-09 } \\ \text { Echinorhynchus baer K. } & \text { P-09 } \\ \text { Echinorhynchus lateralis L. (72) } & \text { P-09 } \\ \text { Echinorhynchus truttae S. (59) } & \text { P-09 } \\ \text { Acanthocephalus echigoensis F. (33) } & \text { P-09 } \\ \text { Dentitruncus truttae S. (53) } & \text { P-01 } \\ \text { Neoechinorhynchus rutilf (O.F.M.) H. (58) } & \text { E-03 } \\ \text { Echinorhynchus lateralis L. (72) } & \text { P-09 } \\ \text { Acanthocephalus dirus (VC.) VC. et T. (3) } & \text { P-09 } \\ \text { Echinorhynchus leidyi V. C. } & \text { P-09 } \\ \text { Echinorhynchus coregoni (L.) in VC. } & \text { P-09 } \\ \text { Rhadinorhynchoides miyagawai F. et M. } & \text { P-03 } \\ \text { Echinorhynchus coregoni (L.) in VC. (83) } & \text { P-09 }\end{array}$

Famille des OSMERIDAE

Acanthocephalus dirus (VC.) VC. et T. (3)

P-09 Echinorhynchus salmonis O.F.M. (5)

P-09

Famille des COREGONIDAE

Echinorhynchus coregoni(L.) in V.C.

Echinorhynchus salmonis O.F.M (46)

P-09

Neoechinorhynchus tumidus V.C. et B. E-03

Echinorhynchus lateralis L. (72) E-09

Neoechinorhynchus tumidus V.C. et B. E-03

Echinorhynchus salmonis O.F. M. (5) P.09

Acanthocephalus clavula (D.) G.-K. et C. (7) E-09

Neoechinorhynchus rutili (O.F. M) H. (58) E-03

Neoechinorhynchus salmonis L.-C. E-03

Famille des THYMALLIDAE

Paracanthocephalus rauschi S.

P.09

Pomphorhynchus laevis (O.F. M.) V.C. (86) P-10

Echinorhynchus cinctulus P. (28) 
Famille des PLECOGLOSSIDAE

Plecoglossus altivelis (Temminck et Schlegel) Acanthocephalus opsariichthydis $\mathrm{Y}$.

SOUS-ORDRE deS OPISTHOPROCTOIDEI

Famille des ARGENTINIDAE

Argentina sphyraena Linné,1758

Breizacanthus golvani $\mathrm{G}$. et $\mathrm{S}$.

Breizacanthus ligur P., O. et D.S.

SOUS-ORDRE des MYCTOPHOIDEI

Famille des CHLOROPHTHALMIDAE

Chlorophthalmus agassizi Bonaparte

Breizacanthus ligur P., O. et D. S

\section{SOUS-ORDRE deS OSTEOGLOSSOIDEI}

Famille des ARAPAIMIDAE

Arapaima gigas (Cuvier,1817) Müller, 1846

Polyacanthorhynchus macrorhynchus (D.) T.(50)

Famille des CLUPISUDIDAE

Clupisudis niloticus (F.) Swainson

(= Heterotis $n$. Ehrenberg )

Esox americanus Gmelin

Esox lucius Linné

Umbra limi (Kirtland)

Brycon hilari (Cuvier et Valenciennes)

Characinidae sp.

Colossoma nigripinnis (Cope)

Hoplerythrinus unitaeniatus (Agassiz)

Hoplias malabaricus (Bloch)

Mylassoma paraguayensis (Norman)

Myletes macropomus Kner

Mylinae so.

Piaractus trachyoomus (Cuvier)
Tenuisentis niloticus (M.) V.C.

E-01

\section{SOUS-ODRE des ESOXOIDEI \\ Famille des ESOCIDAE}

Pilum pilum W.

Acanthocephalus lucii (O.F. M.) L. (94)

Acanthocephalus clavula (D.) G.-K. et C. (15)

Acanthocephalus dirus (V.C.) V.C et T. (3)

P.09

P.09

P.09

P-09

Famille des UMBRIDAE

Neoechinorhynchus limi M. et B.

E-03

Fessisontis tichiganensis A. (2)

\section{ORDRE DES CYPRINIFORMES \\ SOUS-ORDRE deS CHARACOIDEI \\ Famille des CHARACINIDAE}

Echinorhynchus briconi M. F.

P-09

Echinorhynchus gomesi M. F.

Neoechinorhynchus buttnerae G. $\quad$ E-03

Quadrigyrus nickoli S. et $\mathrm{H}$. E-02

Quadrigyrus torquatus V.C. (75) E-02

Quadrigyrus brasiliensis M. F. E-02

Quadrigyrus torquatus V.C. E-02

Neochinorhynchus paraguayensis M. F. (56) E-03

Echinorhynchus salobrensis M. F. E-09

Neochinorhynchus buttnerae G. E-03

Echinorhynchus gomesi M. F. E.09

Echinorhynchus jucundus T. E-09 
Triportheus angulatus (Spix)

Tripotheus paranensis (Günther)
Palliolisentis quinqueungulis M. F. (51)

Palliolisentis quinqueungulis M.F.

Echinorhynchus paranensis M.F.
E-02

E-02

E-09

\section{Famille des ANOSTOMIDAE}

\author{
Caenotropus punctatus (Müller et Troschel) \\ (= Chilodus p.) \\ Curimata elegans Steindachner \\ Prochilodus platensis \\ Prochilodus reticulatus Steindachner \\ Prochiladus scrofa Steindachner
}

Octospiniferoides australis $\mathrm{S}$. et $\mathrm{H}$.

E-03

Neoechinorynchus spectabilis M. F.

Neoechinorhynchus australis V. C. (35)

Neoechinorhynchus prochilodorum N. et T.

Neoechinorhynchus curemai N. (61)

E-03

E-03

E-03

E-03

Famille des CITHARINIDAE

Citharinus distichoides Oken

Neoechinorhynchus africanus T.

E-03

\section{SOUS-ORDRE des CYPRINOIDEI \\ Famille des CYPRINIDAE}

Argyreus cataractae

Pomphorhynchus bulbocolli (L.)n V. C. (71)

P-10

(= Rhinichthys c.)

Barbus barbus (Linné,1758)

Barbus meridionalis Risso

Barbus stigma (Cuvier et Valenciennes)

Barbus ticto (Hamilton-Buchanan)

Catla catla

Carassius auratus Linné,1758

Carassius auratus gibelio

Carassius carassius (Linné,1758)

Chanodichthys macropus

Chondrostoma nasus (Linné,1758)

Cirmina cuipa

(= Grithia c ???)

Cyprinus carpio Linne, 1758

Diptychus maculatus (Steindachner)

Gnathopogon elongatus (Temminck et Schlegel)

Gymrocypris przewalskii

Hampa'a macrolepidota van Hasselt

Hemibarbus barbus

Hybognathus hankinsoni

Hybopsis gracilis

Hypcofitlialmichihys molitrix Cuv. ei Val.

Labeo gonius (Hamilton-Buchanan)

Laboo rotita (Hamilton-Buchanan)

Leuciscus hakonensis Günther
Pomphorhynchus laevis (Z. in O.F. M.) V.C. (86) P-10

Acanthocephalus haranti $\mathrm{G}$. et $\mathrm{O}$.

P.09

Acanthogyrus (Acanthosentis) holospinus (S.) D. et G.

Acanthogyrus (Acanthosentis) dattai (P.) D. et G.

Acanthogyrus (Acanthosentis) dattai (P.) D. et G.

E-01

Acanthogyrus (Acanthosentis) tripathii $\mathrm{R}$.

Pomphorhynchus bulbocalli (L.) in V.C.

Acanthocephalus dirus (V.C.) V.C. et T.

Neochinorhynchus rutili (O.F. M.) H. (1)

Cathayacanthus exilis (V.C.) G.

Pomphorhynchus bulbocolli (L.) in_V.C. (71)

Acanthocephalus dirus (V.C.) V.C. et T.

Micracanthorynchina motomurai (H.) H.L. W. (34)

Neoechinorhynchus rutili (O.F. M.) H. (85)

Pallisentis ophiocephali (T.) B. (31)

E-02

E-02

E-10

P.09

E-03

P.03

P.10

P.09

P-03

E-03

E-02

Acanthocephalus dirus (V.C.) V.C. et T. (2)

P-09

Pomphorhynchus bulbocolli (L.) in V.C. (71)

P.10

E-03

Acanthocephalus opsariichthydis $\mathrm{Y}$.

P.09

E-03

Neoechinorhynchus quinghaiensis L.-Q., B. et T.

E-02

P.09

Acanthocephalus opsariichthydis $\mathrm{Y}$.

Neoechinorhynchus rutili (O.F. M.) H. (71)

Pomphorhynchus bulbocolli (L.) in V. C. (71)

Acanthogyrus (Acanthosentis) multispinus W.

E-03

P. 10

E-02

E-02

E-03

E-02

E-02

P-09 
Leuciscus leuciscus Linné,1758

Leuciscus walecki

Nocomis asper Lachner et Jenkins Notemigonus crysoleucas (Mitchill)

Notropis cornutus (Mitchill)

Notropis emiliae (Hay)

Notropis pislbryi Fowler

Notropis spilopterus

Notropis umbratilis

Opsarichthys uncirostris

Parabramis pekinensis

Phoxinus steindachneri (Sauvage)

Pimephalus promelas

Pseudaspius leptocephalus

Pseudorasbora parva

Ptychocheilus orgonensis (Richardson)

Richardsonius batteatus (Richardson)

Rutilus rutilus (Linné, 1758)

Semotilus atromaculatus (Mitchill)

Schizopygopsis koslowi Herzenstein Schizopygopsis stoliczkae Steindachner Schizothorax esocinus Heckel

Schizothorax planifrons Heckel

Schizothorax sinuatus

( $=$ Oreinus s.)

Schizothorax sp.

Squalius cephalus (Linné,1758)

Tinca linca (Linné,1758)

Tribolodon hakonensis (Günther)

Varicorhinus sevangi (Filippi)

Zacco platypus

Zacco temmincki
Acanthocephalus clavula (D.) G.-K. et C. (13)

Pomphorhynchus laevis (Z.in O.F.M.) V.C. (76)

P-09

Pseudorhadinorhynchus leuciscus (K.et P.) G.

P.10

.

Pomphomynchus lucyi W.et R.

Neoechinorhynchus notemigoni D. (11)

Acanthocephalus dirus (V.C.) V.C. et T. (3)

Pomphortynchus lucyi W.et R.

Acanthocephalus tahlequahensis O.et B.

Acanthocephalus dirus (V.C.) V.C. et T. (2)-(3)

Acanthocephalus dirus (V.C.) V.C. et T. (2)

Acanthocephalus opsariichthydis $\mathrm{Y}$.

Pseudorhadinorhynchus pseudaspii A. et D.-A. (1)

Pseudorhadinorhynchus markewitchi A. et D. A. (1)

Acanthocephalus opsariichthydis $\mathrm{Y}$.

Neoechinorhynchus rutili (O.F.M.) H. (71)

Acanthocephalus dirus (V.C.) V.C. et T. (3)

Pseudorhadinorhynchus pseudaspii A. et D.-A.

Pseudorhadinorhynchus markewitchi A. et D.-A.

Acanthocephalus opsariichthydis $\mathrm{Y}$.

Neoechinorhynchus salmonis C.

Pomphorhynchus bulbocolli (L.) in V.C. (73)

Acanthocephalus clavula (D.) G.-K. et C. (7)

Acanthocephalus dirus (V.C.) V.C. et T. (2)

Neoechinorhynchus saginatus V.C. et B. (78)

Paulisentis fractus V.C. et B.

Paulisentis missouriensis $\mathrm{K}$.

Echinorhynchus alpinus v.L.

Acanthocephalus kashmirensis D.

Neoechinorhynchus yalei (D.) K.

Neoechinorhynchus devdevi (D.) $\mathrm{K}$.

Neoechinorhynchus manasbalensis $\mathrm{K}$.

P.10

E-03

P.09

P-10

P.09

P.09

P.09

P.09

P-01

P-01

P-09

E-03

P.09

P-01

P.01

P.09

E-03

P.10

P.09

P.09

E. 03

[-03

$\mathrm{L}-03$

P.09

P.09

E-03

E-03

E-03

Echinorhynchus orientalis $\mathrm{K}$.

P.09

Pomphorhynchus laevis (Z.in O.F.M.) V.C. P-10

Acanthocephalus graciliacanthus $M$.

P.0.3

Pseudorhadinorhynchus leuciscus (K.et P.) G. (52)

Pomphorhynchus kostylewi P.

P.01

[F.1.

Acanthocephalorhynchoides cholodkowskyi (K.) W. (89)

Acanthocephalus opsariichthydis $\mathrm{Y}$.

0.63

Micracanthorhynchina molomurai (H.) H.L.W.

Micracanthorhynchina motomurai (H;) H.L.W.

P.0.2

P.03

Micracanthorhynchina dakusuiensis (H.) H.L.W. P-03

Neoechinorhynchus formosanus $(\mathrm{H}$.) $\mathrm{Y}$.

E-03

Famille des CATOSTOMIDAE

Neoechinorhynchus prolixus V.C.et T.

Pomphorhynchus lucyi W.et R.

F.10

Neoechinorhynchus carpiodi D.

E. 03

Neoechinorhynchus prolixus V.C. et T. 
Catostomus commersoni (de Lacépède)

Catostomus macrocheilus Girard

Catostomus occidentalis Ayres

Erimyzon oblongus

Erimyzon sucetta (de Lacépède)

Hypentelium nigricans

Ictiobus bubalis (Rafinesque)

ktiobus sp.

ktiobus sp.

Minytrema melanops (Rafinesque)

Moxosioma erythrurum

Moxosioma macrolepidotus

Crossostoma davidi Sauvage

Hymenophysa curta

Misgurnus anguillicaudatus (Cantor)

Misgurnus fossilis (Linné, 1758)

Nemacheilus barbatula toni

Nemacheilus kashmirensis Hora

Nemacheilus stoliczkae Steindachner

Nemacheilus vittatus (Heckel)
Acanthocephalus dirus (V.C.) V.C. et T. Pomphorhynchus bulbocolli (L.) in V.C. Octospinifer macilentus V.C.

Neoechinorhynchus prolixoides B.

Neoechinorhynchus cristatus L.

Neoechinorhynchus crassus V.C. (49)

Neoechinorhynchus cristatus $L$.

Neoechinorhynchus venustus $\mathrm{L}$.

Pomphorhynchus bulbocolli (L.) in V.C. (71)

Octospinifer torosus V.C. et $\mathrm{H}$.

Neoechinorhynchus prolixoides B.

Pomphorhynchus lucyi W.et $\mathrm{R}$.

Neoechinorhynchus robertbraveri A.

Acanthocephalus dirus (V.C.) V.C. et T. (2)

Leptorhynchoides thecatus (L.) K. (47)

Neoechinorhynchus strigosus V.C.

Neoechinorhynchus australis V.C.

Acanthocephalus dirus (V.C.) V.C. et T.

Pomphorhynchus lucyi W.et R.

Acanthocephalus dirus (V.C.) V.C. et T.

Pomphorhynchus bulbocolli (L.) in V.C. (71)

P-09

P.10

E-03

E-03

E-03

E-03

E-03

E-03

P.10

E-03

E-03

P-10

E-03

P-09

P.02

E-03

E-03

P-09

P-10

P-09

P.10

\section{Famille des COBITIDAE}

Neoechinorhynchus wuyiensis W.P.Q

E-03

Acanthocephalus opsariichthydis $\mathrm{Y}$.

Acanthocephalus opsariichthydis $\mathrm{Y}$.

Tenuiproboscis misgurni $\mathrm{Y}$.

Sachalinorhynchus skrjabini K.et P.

Pomphorhynchus kashmirensis $\mathrm{K}$.

Neoechinorhynchus rutili in Datta, 1936

Neoechinorhynchus rutili in Datta,1936

P-09

P-09

P.10

P-06

P-10

E-03

E-03

\section{SOUS-ORDRES deS SILUROIDEI \\ Famille des ARIIDAE}

Rhadinorhynchus arii W.

P-03

Indorhynchus indicus (T.) G.

P-01

Gorgorhynchus medius (L.) C.

P-03

Gorgorhynchus medius (L.) C.

P-03

Indorhynchus indicus (T.) G.

P-01

\section{Famille des BAGRIDAE}

Acanthogyrus (A.) antespinus (V.et D.) D.et G.

Rhadinorhynchus arii W. P-03

Famille des DORADIDAE

$\begin{array}{ll}\text { Neoechinorhynchus pterodoridis T. } & \text { E-03 } \\ \text { Paracavisoma impudica (D.) VK. } & \text { P-04 } \\ \text { Paracavisoma impudica (D.) VK. } & \text { P-04 }\end{array}$

Paracavisoma impudica (D.) VK.
Centrochir granulosus (Valenciennes) (= Petrodoras c.)

Doras costatus

Pseudodoras niger (Valenciennes)

(=Rhinodoras $n$.) 
Ictalurus furcatus

ktalurus melas

( $=$ Ameiurus m.)

Ictaluns meridionalis

Ictalurus natalis (LeSueur)

Ictalurus punctatus

Pilodictis olivaris

\section{Famille des ICTALURIDAE}

Pomphorhynchus bulbocolli (L.) in V.C. (71) Acanthocephalus dirus (V.C.) V.C. et T. (2)

P.10

P-09

Neoechinorhynchus golvani S.-M. (68) E-03

Pilum pilum $\mathrm{W}$.

Acanthocephalus dirus (V.C.) V.C. et T. (2)

Acanthocephalus dirus (V.C.) V.C. et T. (2)-(3)

P.09

P.09

P.09 Pomphorhynchus bulbocolli (L.) in V.C. (71)

P.10

Famille des LORICARIIDAE

Gorytocephalus elongorchis T.

E-03

Gorytocephalus plecostomorum N.et T.

E-03

Famille des MOCHOCIDAE

Synodontis membranaceus (Et.Geoffroy, 1809) Pallisentis golvani T.et V. (54)

Famille des PANGASIIDAE

Pangasius pangasius (Hamilton-Buchanan)

Cleaveius prashadi (D.) G.

P-03

Famille des PLOTOSIDAE

Plotosus anguillaris de Lacépède

Plotosus caninus (Hamilton-Buchanan)

Longicollum alemniscus F.et $M$.

P.10

Paracanthocephaloides plotosi (Y.) G.

P.07

Arhythmacanthus septacanthus $\mathrm{S}$.

P-07

Famille des SCHILBEIDAE

Clupisoma garua (Hamilton-Buchanan)

Pallisentis garuai (S., S.et G.) J.et G

E-02

Famille des SILURIDAE

Parasilurus asotus (Linné)

Acanthocephalus minor $Y$

Echinorhynchus parasiluri $\mathrm{F}$.

Pallisentis celatus (V.C.) $\mathrm{H}$.

Wallago attu

Pallisentis magnum S.et $\mathrm{B}$.

P.09

P.09

E-02

E-02

\section{ORDRE DES ANGUILLIFORMES}

Famille des ANGUILLIDAE

Anguilla anguilla (Linné,1758)

(=A.vulgaris Shaw, 1803)

Anguilla chrysypa

(=A.rostrata LeSueur)

Anguilla pekinensis

Anguilla reinhardti (Steindachner)
Acanthocephalus clavula (D.) G.-K.et C. P.09

Acanthocephalus haranti G.et $O$.

P.09

P-01

E-03

P.02

E-01

E-02

P.01 


\section{ORDRE DES SYMBRANCHIFORMES}

Famille des FLUTIDAE

\section{ORDRE DES PERCOPSIFORMES}

\section{Famille des APHREDODERIDAE}

Aphredoderus sayanus (Gilliams)

Pilum pilum W.

P.09

Leptorhynchoides aphredoderi B.et B.

P.02

\section{ORDRE DES BELONIFORMES \\ SOUS-ORDRE deS BELONOIDEI \\ Famille des BELONIDAE}

Belone belone (Linné,1758)

Telosentis molini V.C.

P-01

(=B.acus Nybelin,1942 nec de Lacépède)

(=B.vulgaris Fleming,1828)

Strongylura marina (Walbaum)

Tylosurus acus (de Lacépède, 1803)

Tylosurus schismatorhynchus (Bleeker)

Tylosurus strongylurus

Pomphorhynchus lucyi W.et R.

Rhadinorhynchus ornatus V.C.

Neoechinorhynchus tylosuri $Y$.

Longicollum indicum G.et $\mathrm{G}$.

Famille des SCOMBRESOCIDAE

Cololabis saira (Brevoont,1850)

Rhadinorhynchus cololabis Let McC.

P-03

Sous-Ordre des EXOCOETOIDEI

Famille des EXOCOETIDAE

Exocoetus volitans Linné, 1758

Telosentis molini V.C.

P.01

Famille des HEMIRHAMPHIDAE

Hemirhamphus intermedius Hyporhamphus sayori (Temm.et Schleg.)
Micracanthorhynchina hemirhamphi (B.) H.L.W.(42)

Allorhadinorhynchus segmentatus $\mathrm{Y}$.
P-03

P-05

\section{ORDRE DES GASTEROSTEIFORMES}

Famille des GASTEROSTEIDAE

Gasterosteus aculeatus Linné Pungitius pungitius (Linné) (a Pygosteus $p$.)
Neoechinorhynchus rutili (O.F.M.) H. Neoschinorhynchus pungitius $D$.

E.03

\section{ORDRE DES MUGILIFORMES}

Famille des MUGILIDAE

Aldrichetta forsteri (Cuv.et Val.)

Crenimugil crenilabrus (Forsskal)

Liza macrolepis

Liza subviridis (Cuv.et Val.)
Pararhadinorhynchus coorongensis E. Neoechinorhynchus aldrichettae E. Neoechinorhynchus agilis sensu E. (20) Neoechinorhynchus sp. A.,N., AY.et AH. Neoechinorhynchus elongatus $T$. Pseudorhadinorhynchus orissai G.et F.
P.05

E-03

E-03

E-03

E-03

P.01 
360

Mugil cephalus Linné, 1758

Mugil curema Cuv.et Val.

Mugil dussumieri Cuv.et Val.

Mugil parsia Hamilton-Buchanan

Mugil platanus Günther

Mugil tade Forsskal

Mugilsp.

Mugil sp.
Y. J. GOLVAN, I. DE BURON

Pararhadinorhynchus mugilis J.et E.

Pseudorhadinorhynchus srivastavai G.et F.

Neoechinorhynchus agilis (R.) V.C. (90)

Floridosentis elongatus H.L.W. (69)

Floridosentis bravohollisae nom.nov. (10)

Floridosentis pacifica B.-H.

Neoechinorhynchus elongatus T.

Neoechinorhynchus chilkaensis P. (29)

Atactorhynchus mugilis M.F.

(=Floridosentis (?)

Neoechinorhynchus bangoni $\mathrm{T}$.

Atactorhynchus mugilis M.F.

Gracilisentis mugilis G.et $\mathrm{L}$.

Famille des ATHERINIDAE

Telosentis exiguus (VL.) V.C. (44)

Telosentis molini V.C.

P.01

Heterosentis heteracanthus (VL.) G.

\section{ORDRE DES POLYNEMIFORMES}

Famille des POLYNEMIDAE

Galeoides decadactylus (Bloch)

Polynemus heptadactylus Cuv.et Val.

Polynemus octonemus

Polynemus sextarius Bloch et Schneider (= Polydactylus s.)
Rhadinorhynchus atheri (F.) A.

Rhadinorhynchus cadenati (G.et H.) G. (21)

Rhadinorhynchus polynemi G.ret L.

Dolfusentis longispinus (C.et L.) G.

Raorhynchus polynemi T.
P-03

P-03

P-03

P.01

P-03

\section{ORDRE DES CYPRINODONTIFORMES Famille des CYPRINODONTIDAE}

Cyprinodon variegatus Fundulus grandis Baird et Girard Jordanella floridae Goode et Bean

Gambusia affinis

Fitzroyia lineata (Jenyns)
Atactorhynchus verecundus $\mathrm{C}$.

Octospiniteroides chandleri B. (12)

Neoechinorhynchus doryphorus V.C. et $\mathrm{B}$

Famille des POECILIDAE

Octospinfiforoides chandleri B. (12)

Famille des FITZROYIDAE

Wolthugelia matercula M.-G. et D.C.

\section{ORDRE DES GADIFORMES}

Famille des MACRURIDAE

Coelorhynchus coelorhynchus (Risso, 1810)

Breizacanthus ligur P.,O.et D.S

Famille des GADIDAE

Breizacanthus ligur P.,O. et D.S.

P.07

Echinorhynchus yamagutii $\mathrm{G}$.

E-03

E-03

E-03

Gadiculus argenteus Guichenot,1850

Gadus macrocephalus Tilesius 
Gadus morhua Linné,1758

(= G.callerias Linné, 1758 pp.)

Gaidropsarus vulgaris (Cloquet,1824)

(= G.tricirratus de Buen,1934)

Lota lota Linné, 1758

L stella phycis (Temm.ot Schlegel)

Microgadus tomcod (Walbaum)

Phycis blennoides (Brünnich,1768)

Pollachius pollachius (Linné,1758)

Pollachius virens (Linné, 1758)

Theragra chakogramma (Pallas)
Echinorhynchus gadi O.F.M.

Echinorhynchus vancleavei G.

Echinorhynchus calloti $\mathrm{G}$.

Echinorhynchus cinctulus $\mathrm{P}$.

Echinorinynchus rhenanus $\mathrm{G}$.

Echinorhynchus lotellas $\mathrm{Y}$.

Echinorhynchus vancieavei $G$

Breizacanthus ligur P., O. et D.S.

Echinorhynchus armoricanus G.

Echinorhynchus armoricanus $\mathrm{G}$.

Echinorhynchus yamagutii $\mathrm{G}$.

Famille des MERLUCCIDAE

Merluccius sp.

Aspersentis johni

(B.) $\mathrm{C}$.

P-06

\section{ORDRE DES ZEIFCRMES}

Famille des CAFROIDAE

Breizacanthus ligur P.,O et D.S.

P-07

\section{ORDRE DES PEFICIFORMIES \\ SOUS-ORDRE DES FERCOIDEI \\ Famille des LATIDAE}

Lates niloticus Cuvier et Valenciennes, 1828

Paragorgorhynchus chariensis T.

P-03

Famille des SERRANIDAE

Epinephelus akaara (Temminck et Schlegel)

Gorgorhynchus epinepheli (Y.) G.

Neoechinorhynchus sinicus $W$.

E-03

Gorgorhynchus medius (L.) C. (80)

P.03

Mycteroperca apua (Richardson)

Paralabrax humeralis ( Cuvier et Valenc.)

Percichthys melanops (Girard)

Serranus sp.

Stereolepis ischinagi

Pomphorhynchus yamagutii S.et $\mathrm{H}$.

P-03

P-10

P-C4

Cavisoma magnum (S.) V.C.

Echinorhynchus yamagutii $\mathrm{G}$. (90)

P-09

Famille des OLIGORIDAE

Acanthogyrus (Acanthosentis) paucispinus W. E-02 Neoechinorhynchus sinicus $W$.

Goigorhynchus satoi M.

E-03

P.03

Famille des MORONIDAE

Acanthocephalus dirus (V.C.) V.C. et T. (2)

P-09

Pomphorhynchus rocci C.et H.L.W.

P-10

\section{Famille des CENTRARCHIDAE}

Ambloplites rupestris (Rafinesque)

Chaenobryttus coronarius ( Bartram)
P.02

$P-10$

P-09

E-03

E-03 
Lepomis auritus (Linné)

Lepomis cyanellus Rafinesque

Lepomis gibbosus (Linné,1758)

Lepomis gulosus (Cuvier)

Lepomis macrochirus (Ratinesque)

Lepomis marginatus (Holbrook) Lepomis microlophus (Günther) Lepomis punctatus (Valenciennes)

Micropterus dolomieui

Micropterus salmoides (de Lacépède)

(= Huro s.)

Pomoxis nigromaculatus (LeSueur)

Etheostoma punctulatum (Agassiz)

Etheostoma spectabile (Agassiz)

Etheostoma squamiceps

Perca flavescens

Perca fluviatilis Linné, 1758

Percina caprodes (Ratinesque)

Siniperca chuatsi

Stizostedion vitreum

Cestreus regalis

(= Cynoscion r.)
Pilum pilum W.

P.09

Pomphorhynchus lucyi W.et R.

Leptorhynchoides thecatus (L.) K. (79)

Acanthocephalus dirus (V.C.) V.C. et T. (2)

Eocollis arcanus V.C. (57)

Leptorhynchoides thecatus (L.) K. (79)

Acanthocephalus dirus (V.C.) V.C. et T. (2)

Fessisentis friedi N. (32)

Piumpilum W.

Pilum pilum $W$.

Eocollis arcanus V.C.

Acanthocephalus dirus (V.C.) V.C.et T. (2)

Pomphorhynchus lucyi W.et R

Pomphorhynchus lucyi W.et R.

Pomphorhynchus lucyi W.et R.

Pilum pilum W.

Leptorhynchoides thecatus (L.) K. (16)

Neoechinorhynchus cylindratus (V.C.) V.C. (88)

Leptorhynchoides thecatus (L.) K. (16)

Acanthocephalus dirus (V.C.) V.C. et T. (2)

Eocollis arcanus V.C.

Acanthocephalus dirus (V.C.) V.C. et T. (2)

P.10

P-02

P.09

E-03

P.02

P.09

P-04

P-09

P.09

E-03

P-09

P.10

P-10

P.10

P-09

P.02

E-03

P-02

E-03

P.09

\section{Famille des PERCIDAE}

Acanthocephalus tahlequahensis O.et B. (3) Acanthocephalus tahlequahensis O.et B. (3) Acanthocephalus dirus (V.C.) V.C. et T.

Leptorhynchoides thecatus (L.) K. (47)

Acanthocephalus dirus (V.C.) V.C. et T. (2)

Acanthocephalus lucii (O.F.M.) L. (45)

Acanthocephalus clavula (D.) G.-K. et C. (7)

Pomphorhynchus bulbocolli ((L.) in V.C.

Hebesoma violenteum V.C.

(= (?) Neoechinorhynchus v.)

Acanthocephalus dirus (V.C.) V.C. et T. (2)

P-09

P.09

P.09

P.02

P.09

P.09

P.09

P.10

E-03

P-09

Famille des OTOLITHIDAE

Dollfusentis chandleri $\mathrm{G}$.

P-01

Famille des RACHYCENTRIDAE

Rachycentron canadum (Linné, 1766)

(= Elacate nigra (Bloch, 1793) Günther,1860)

Serrasentis sagittifer (L.) L. (25)

P.03

Serrasentis nadakali $\mathrm{G}$.et $\mathrm{N}$
Caranx hippos (Linné, 1758)

Caranx latus Agassiz, 1829

Caranx mertensi Cuvier el Valenc.

Caranx sp.

Seriola quinqueradiata

Seriola zonata (Mitchill,1815)

(= S.lalandi Valenciennes,1833)
Famille des CARANGIDAE

Gorgorhynchoides bullocki C.et M. (67)

Filisoma bucerium V.C. (66)

Gorgorhynchoides bullocki C.et M. (67)

Rhadinorhynchus carangis $\mathrm{Y}$. (20)

P.04

P.07

P.03

P-07

Gorgorhynchoides bullocki C.et M. (67)

P.03

Gorgorhynchoides lintoni C.et M. 
Trachurus japonicus (Temminck et Schlegel)

Rhadinomynchus trachuri $\mathrm{H}$.

Pomphorhynchus francoisae G.

Trachynotus ovatus (Linné, 1758)

(- Lichia glauca Cuvier,1832)

Zonichthys falcatus (Linne, 1758)

Gorgorhynchoides lintoni C.et $\mathbf{M}$.

P-10

(- Trachynotus f.)

Serrasentis sagittifer (L.) L.

Famille des LOBOTIDAE

Lobotes surinamensis (Bloch,1790)

Dolfusentis chandleri G. (80)

P.01

Famille des LUTJANIDAE

Lutjanus johni (Bloch)

Lutjanus russelli

Longicollum lutiani J.et $\mathbf{G}$.

P.10

Longicollum alemniscus $(H$.) $Y$.

P.10

Famille des NEMIPTERIDAE

Nemipterus japonicus

Femogibbosus assi P.

P-04

Famille des LEIOGNATHIDAE

Leiognathus equulus (Forsskal)

Leiognathus splendens (Cuvier)

Raorhynchus schmidti G.et N.

P-03

Goacanthus panajiensis G.et $\mathrm{J}$.

Famille des POMADASYIDAE

Anisotremus virginicus (Linné, 1758)

Dolfusentis longispinus (C.et L.) G.

P.01

Haemulon melanorum (Linné,1758)

Pomadasys crocro

Pomadasys hasta Günther

Dolfusentis chandleri G. (65)

Dolfusentis bravoae S.M.

P-01

Acanthocephalus hastae B.

P.01

P.09

Famille des SCIAENIDAE

\section{Aplodinoptus grunniens}

Aplodinotus simer

Johnius goma (Tanaka)

Leiostomus xanthurus de Lacépède, 1803

Micropogon undulatus (Linné)

Menticirrhus americanus (Linné)

Menticirrhus undulatus (Girard)

Plagioscion squámossissimus (Heckel)

Pseudoscion diacanthus de Lacépède

Roncador stearnsi (Steindachner)

Umbrina roncador Jordan et Gilbert

Mullus surmuletus Linné,1758

Upeneichthys porosus (Cuvier et Valenc.)

Pagrosomus unicolor (Quoy et Gaimard) Sparus macrocephalus (Basilewski,1855) (nec maculocephalus)
Acanthocephalus dirus (V.C.) V.C. et T. P.09

Fessisentis fessus V.C.

Pomphorhynchus bulbocolli (L) in V.C. (71)

Acanthocephalus dirus (V.C.) V.C. et T. (3) P.09

Neoechinorhynchus goma $Y$. E-03

Telosentis tenuicornis V.C. P-01

Telosentis tenuicornis V.C. P-01

Illiosentis furcatus V.C. et $\mathrm{L}$ P.01

Illiosentis cetratus V.C.

Rhadinorhynchus plagioscionis T. P.03

Neoechinorhynchus johni Y. (39) E-03

Illiosentis cetratus V.C. P-01

Illiosentis cetratus V.C. $\quad$ P-01

Famille des MULLIDAE

Breizacanthus chabaudi G. P-07

Breizacanthus irenae G. $\quad$ P.07

Euzetacanthus simplex (R.) G.et H. P.07

lliosentis edmondsi G. (19) P-01

Famille des SPARIDAE

Longicollum pagrosomi $\mathrm{Y}$. P-10

Longicollum alemniscus (H.) Y. $\quad$ P-10 


\section{Famille des SCORPIDAE}

Medialuna sp.

Kyphosus elegans (Peters)
Tegorhynchus pectinarius V.C.

\section{Famille des KYPHOSIDAE}

Filisoma bucerium V.C.

Famille des SCATOPHAGIDAE

Scatophagus argus (Cuvier et Valenciennes)

Filisoma indicum V.C. (38)

Filisoma scatophagusi D.et $\mathrm{S}$.

P-04

Longicollum alemniscus (H.) Y. (23)

Famille des CICHLIDAE

Asquidens pulchor (Giil)

Cichlasoma fonestratum

Cichlasoma meeki

Cichlasoma pearsei

Cichlasoma urochthaimus

Crenicichla geayi Pellegrin

Geophagus brasilignsis

Petenia spisndida

Tilapia houdeloti

Tilapia lidolo
Pandosentis iracundus V.C.

Neoechinorhynchus goivani S.M.

Noovchinornynchus golvani S.M.

Nooochinorhynchis golvani S.M (68)

Nooochinorhynchus golvani S.M. (68) E-03

Pandoseritis iracundus V.C.

Neoechinorhynchus paraguayensis M.F. (60) E-03

Nooechincrhynchus golvani S.M. (68) E-03

Acanthogj'rus (Acanthosentis) tilapiae (B.)D.etG. (54) E-02

Acanthogyrus (Acanthosentis) tilapiae (B.)D.et G. E-02

\section{SOUS-ORDRE CES LABROIDEI \\ Famille des EMIE:OTOCIDAE}

Ditrema temmincki Bleeker

Hospilabrus reticulatus (Cuv.et Val.)

Chromis marginatus

ou C. multilineata Guichenot, 1853
Rhadinorhynchus ditrematis $Y$. Rhadinorhynchus carangis $\mathrm{Y}$.

P-03

P-03

Famille des LABRIDAE

Tegorhynchus brevis V.C.

P-01

Famille des POMACENTRIDAE

Fseudocavisoma chromitidis (C.et Q.) G.et H.

P.04

\section{SOUS-ORDRE DES TRACHINOIDEI \\ Famille des CHAENICHTHYIDAE}

Chaenichthys rhinoceratus Richardson

Chaenocephalus aceratus (Lönnberg,1906)

Champsocephalus gunnari Lönnberg,1905

Chionodraco rastiospinosus de Witt, 1979
Aspersentis austrinus V.C. (92)

Aspersentis austrinus V.C. (92)

Metacanthocephalus dalmori Z.

Metacanthocephalus johnstoni Z.

Metacanthocephalus dalmori Z.

Famille des.BATHYDRACONIDAE 
Parachaenichthys georgianus Fischer

Metacanthocephalus campbelli (L.et A.) G. (93)

\section{Famille des NOTOTHENIIDAE}

Eleginops maclovinus (Cuvier et Valenc.) Nctothenia acuta Günther Notothenia coriiceps Richardson

Notothenia coriiceps neglecta

Notothenia cyanobrancha Richardson Nototheria gibberifrons

Notothenia myzops Günther Notothenia nudifrons

Notothenia rossi Richardson

Notothenia rossi marmorata

Notothenia sp.

Trematomus bernacchii Boulenger,1902

Trematomus borchgrevinski

Trematomus centronotus

Trematomus eulepidotus

Trematomus hansoni Boulenger, 1902

Trematomus newnesi Boulenger,1902
Hypoechinorhynchus magellanicus S.

Aspersentis austrinus V.C.

Aspersentis austrinus V.C. (18)

Metacanthocephalus campbelli (L.et A.) G.

Metacanthocephalus rennicki (L.et A.) Z.

Metacanthocephalus johnstoni Z.

Aspersentis austrinus V.C.

Echinorhynchus nototheniae $\mathbf{Z}$.

Heterosentis heteracanthus (vL.) V.C.

Metacanthocephalus johnstoni Z.

Metacanthocephalus dalmori Z.

Aspersentis austrinus V.C. (18)

Aspersentis austrinus V.C.

Heterosentis heteracanthus (VL.) V.C.

Metacanthocephalus johnstoni Z.

Metacanthocephalus dalmori $\mathbf{Z}$.

Aspersentis austrinus V.C.

Asparsentis austrinus V.C.

Metacanthocephalus johnstoni Z.

Metacanthocephalus dalmori Z.

Aspersentis austrinus V.C.

Asporsentis megarhynchus (vL.) G.

Rhadinorhynchus wheeleri $\mathrm{B}$.

Aspersentis austrinus V.C.

Heterosentis heteracanthus (vL.) V.C.

Metacanthocephalus johnstoni $Z$.

Metacanthocephalus dalmori $\mathbf{Z}$.

Aspersentis austrinus V.C.

Metacanthocephalus dalmori Z.

Metacanthocephalus johnstoni Z. (91)

Metacanthocephalus rennicki (L.et A.) Z. (91)

Metacanthocephalus campbelli (L.et A.) G. (92)

Melacanthocephalus campbelli (L.et A.) G. (92)

Metacanthocephalus campbelli (L.et A.) G. (92)

Metacanthocephalus rennicki (L.et A.) Z. (93)

Aspersentis austrinus V.C. (93)

Echinorhynchus nototheniae Z. (93)

Metacanthocephalus johnstoni Z.

Aspersentis austrinus V.C. (91)

Metacanthocephalus johnstoni $\mathrm{Z}$.

Melacanthocephalus dalmori Z.
P.06

P.06

P-02

P.02

P.02

P-06

P.09

P.06

P-02

P-02

P.06

P.06

P.06

P-02

P-02

P-06

P-06

P-02

P-02

P-06

P-06

P-06

P-06

P.06

P-02

P-02

P-06

P.02

P-02

P.02

P-02

P-02

P-02

P.02

P-06

P.09

P.02

P-06

P.02

P.02

\section{SOUS-ORDRE DES CALLIONYMOIDEI Famille des CALLIONYMIDAE}

Callionymus calauropomus Callinnymus phaeton Günther,1861
Hypoechinorhynchus alaoopis $\mathrm{Y}$. Breizacanthus ligur P.,O.et D.S.
P-08

P-07 


\section{SOUS-ORDRE DES BLENNIOIDEI}

Famille des BLENNIIDAE

Blennius pavo Risso, 1810

Golvanacanthus blennii P.et O.

F.65

Famille des ZOARCIDAE

Pachycara obesa Zugmayer,1911

Rhigophila dearboni

Echinorhynchus abyssicola Dolifus, 1931

P-09

Metacanthocephalus campbelli (L.et A.) Z.

P-02

SOUS-ORDRE DES OPHIDIOIDEI

Famille des BROTULIDAE

Neobythites macropus Günther

Yamagutisentis neobythitis (Y.) G.

Yamagutisentis rhinoplagusiae (Y.) G.

\section{SOUS-ORDRE DES ACANTHUROIDEI \\ Famille des ACANTHURIDAE}

Acanthurus bahianus Castelnau, 1855 Acanthurus chirurgus (Bloch,1787)

Acanthurus coeruleus Bloch et Schneider Acanthurus monroviae Steindachner Acanthurus strigosus

Xesurus scalprum (Cuvier et Valenc.)
Acanthogyrus (A.) acanthuri C.et $Q$. Acanthogyrus (A.) acanthuri C.et $Q$. Acanthogyrus (A.) acanthuri $\mathrm{C}$. et $\mathrm{Q}$. Acanthogyrus (A.) acanthuri C.et $Q$. Cavisoma magnum (S.) V.C.

Noorhadinorhynchus aspinosus (F.et M.) Y.
E-01

E-01

E-01

E-01

P-04

P.04

SOUS-ORDRE DES SIGANOIDEI

Famille des SIGANIDAE

Siganus lineatus (Cuvier et Valenc.) Siganus oramin (Bloch et Schneider) (= Amphacanthus 0.)

Siganus rivulatus Forsskal,1775

Sclerocollum robustum (E.) S.et $P$

P.03

Diplosentis amphacanthi T.et M.

Sclerocollum rubrimaris $S$.et $P$. (4)

Sclerocollum rubrimaris S.et $\mathrm{P}$

P.05

P.03

F.0 $0^{n}$

\section{SOUS-ORDRE DES SCOMBROIDEI}

Famille des SCOMBRIDAE

Rhadinorhynchus seriolae (Y.) G. (37)

P.03

Famille des THUNNIDAE

Euthynnus (Katsuwonus) pelamys (Linné,1758 Rhadinorhynchus cadenati (G.et H.) G. (77)

P-03

(= Thunnus vagans Lesson,1826)

Rhadinorhynchus ornatus V.C. (26)

P-03

Rhadinorhynchus katsuwonis $\mathrm{H}$.

P.03

Rhadinorhynchus vancleavei $\mathrm{G}$.

P.03

Rhadinorhynchus meyeri (H.) G.

Rhadinorhynchus terebra (R.) T. (17)

P.03

P.03

Euthynnus quadripunctatus (Et.Geoffroy, 1817)

Rhadinorhynchus vancleavei $\mathrm{G}$.

P-03

Rhadinorhynchus johnstoni G. (41)
( $=$ E.alliteratus Jordan el Gilbert, 1883)

Thunnus maccoyi

\section{Famille des SCOMBEROMORIDAE}




\section{SOUS-ORDRE DES STROMATOIDEI \\ Famille des CENTROLOPHIDAE}

Palinurichthys perciformis

Stromateus cinereus Day Siromateus sinensis Day

Dolfusentis chandleri $\mathbf{G}$. ou Dolfusentis longispinus (C.et L.) G.

\section{Famille des STROMATEIDAE}

Pseudorhadinorhynchus cinereus G. et N. P-01

Pseudorhadinorhynchus mujibi G.et N

P.01

\section{SOUS-ORDRE DES OPHIOCEPHALOIDEI}

Famille des OPHICEPHALIDAE

Cphiocephalus argus

Pallisentis umbellatus V.C.

$P$ allisentis allahabadi A.

E-02

Ophiocephalus marulius (Hamilton-Buchanan)

Pallisentis ophiocephali (T.) B.

Pallisentis nagpurensis (B.) B. (38)

E-02

Ophiocephalus punctatus (Bloch)

Ophiocephalus striatus (Bloch)

Pallisentis panadei R.

Pallisentis gaboes (MC.) V.C.

Pallisentis nagpurensis (B.) B. (22)

Pallisentis ophiocephali (T.) B.

\section{SOUS-ORDRE DES GOBIOIDEI \\ Famille des ELEOTRIDAE}

Mogurnda obscura (Temminck et Schlegel)

Acanthocephalus minor $\mathrm{Y}$.

Famille des GOBIIDAE

Chaenogobius annularis uranotaenia (Hilgendorf) Gillichthys mirabilis Cooper

Gobius niger Linné, 1758

Zosterisessor ophiocephalus (Pallas, 1811)

(= Gobius tota Valenciennes)
Acanthocephalus opsariichthydis $\mathrm{Y}$.

Microsentis wardae M.et M.

Acanthocephaloides propinquus (D.) M. (13)

Acanthocephaloides propinquus (D.) M. (13)

Acanthocephaloides incrassatus (M.) M. (44)
P.09

E-03

P.07

P.07

P.07

\section{Famille des PERIOPHTHALMIDAE}

Periophthalmus papilio

Acanthogyrus (Acanthosentis) papilio T.et V. (54)

\section{SOUS-ORDRE DES SCORPAENOIDEI}

Famille des SCORPAENIDAE

Holicolenus dactylopterus (Delaroche,1809) Scorpaena neglecta Temminck et Schlegel Sebastes marinus (Linné,1758)

(= S.nonegicus Cuvier, 1829)

Sebastichthys oblongus (Günther)

Sebastiscus marmoratus (Cuvier et Valenc.)

Sebastolobus alascanus
Breizacanthus ligur P.,O.et D.S.

Micracanthorhynchina cynoglossi W.P.

Echinorhynchus (?) Cestodicola vL.

Pomphorhynchus sebastichthydis $\mathrm{Y}$.

Yamagutisentis neobythitis (Y.) G.

Echinorhynchus sebastolobi $\mathrm{K}$.
P-07

P-09

P-10

P.09

P.09

Famille des TRIGLIDAE

Eurrigla gurnardus (Linné, 1758)

Triglopterus lastoviza (Brünnich,1768)

(= Trigla adriatica Gmelin,1789)
Euzetacanthus simplex (R.) G.et H. 
Famille des CONGIOPODIDAE

Famille des HEXAGRAMMIDAE

Hexagrammos otakii

Cottus bairdi Girard

Cottus cognatus Richardson

Cottus gobio Linné

Cottus pollux Günther

Cyclopterus lumpus Linné, 1758

Liparis owstoni

(= Cyclogaster 0. )
Filisoma japonicum F.et M. (23)

P.04

\section{SOUS-ORDRE DES COTTOIDEI}

Famille des COTTIDAE

Acanthocephalus dirus (V.C.) V.C. et T. (2)

Acanthocephalus dirus (V.C.) V.C. et T. (3)

P.09

Acanthocephalus clavula (D.) G.-K. et C. (7)

P.09

Echinorhynchus cotti $Y$.

Famille des CYCLOPTERIDAE

Echinorhynchus gadi in Lundström, 1942 .

P-09

Echinorhynchus salmonis in_Lundström,1942

Famille des LIPARIDAE

Echinorhynchus yamagutii G. (90)

P-09

\section{ORDRE DES PLEURONECTIFORMES \\ SOUS-ORDRE DES PSETTODOIDEI \\ Famille des PSETTODIDAE}

Psettodes eurumei (Bloch et Schneider)

Serrasentis fotedari G.et $F$.

\section{SOUS-ORDRE DES PLEURONECTOIDEI Famille des BOTHIDAE}

Arnoglossus laterna (Walbaum,1792)

Acanthocephaloides geneticus d3.,R.et M.

Famille des PARALICHTHYIDAE

Pseudorhombus triocellatus (Günther)

Raorhynchus schmidti G.et N.

P-03

\section{Famille des PLEURONECTIDAE}

Alaeops plinthus Jordan et Starks

Hippoglossoides platessoides (Fabricius, 1790)

Hippoglossus hippoglossus (Linné, 1758)

Lepidopsotta bilineata (Ayres)

Liopsetta obscura

Platichthys stellatus (Pallas)

Pleuronichthys cornutus (Temminck\&Schlegel)

Pseudoplouronectes amoricanus (Walbaum.)

Scophthalmus aquosus (Mitchill; 1815)
Hypoechinorhynchus alaeopis Y.

Echinorhynchus laurentianus R.

Echinorhynchus laurentianus R.

Echinorhynchus lageniformis $\mathrm{E}$.

Metacanthocephalus ovicephalus (Z.) G.

Echinorhynchus lageniformis E. (63)

Metacanthocephalus pleuronichthydis $Y$.

Echinorhynchus laurentianus R.

Echinorhynchus laurentianus $\mathrm{R}$.
P.08

P-09

P-09

P.C9

P-02

P.09

P-02

P.09

P-09

Famille des RHOMBOSOLEIDAE 


\section{SOUS-ORDRE DES SOLEOIDEI \\ Famille des SOLEIDAE}

Barnardichthys fulvomarginatus (Gilchrist) Solea bleekeri Boulenger, 1898

Solea impar Bennett, 1831

Solea nasuta (Pallas, 1811)
Longicollum chabanaudi D.et G.

Rhadinorhynchus capensis $\mathrm{B}$.

Solearhynchus soleae (P.) dB.et M.

Acanthocephaloides propinquus (D.) M. (44)

Acanthocephaloides kostylewi M.

Famille des ACHIRIDAE

Neoechinorhynchus roseum S.M.
P.10

P.03

P-09

P.07

P.07

Achirus mazatlanus

Famille des CYNOGLOSSIDAE

Cynoglossus robustus Günther

Micracanthorhynchina cynoglossi W.P.Q.

P-03

Cynoglossus zanzibarensis Norman, 1939

Paracanthocephaloides chabanaudi (D.) G.

P.07

Paraplagusia guttata

Arhythmacanthus paraplagusiarum $\mathbf{N}$.

P.07

Rhinoplagusia japonica (Temminck ot Schlegel)

Yamagutisentis rhinoplagusiae (Y.) G.

P.09

Famille des SYNAPTURIDAE

Synaptura orientalis

Echinorhynchus meyeri G.et N.

P-04

\section{ORDRE DES MASTACEMBELIFORMES}

Famille des MASTACEMBELIDAE

Mastacembeius aculeatus

(= Rhy'nchobdella a)

Mastacemboius arriatus

Miichthys mi-iuy (Basilewsky)

poisson d'origine inconnue
Pallisentis basiri F.

Raosentis thapari R.

E-02

POISSONS incertae sedis

Centrorhynchus (???) sinicus W.

Acanthocephalus petersi Tadros, 1966

P-09

\section{Discussion}

\section{1 - Du point de vue des Poissons hôtes définitifs}

La première remarque que l'on doit faire est qu'il ne semble pas exister de Cyclostomes ou de Sélaciens chez lesquels les femelles d'Acanthocéphales puissent atteindre leur pleine maturité. L'Echinorhynchus (s. l.) charchariae Linton, 1891 proviendrait d'Odontaspis taurus (Rafinesque, 1810) (= Carcharias littoralis Mitchill, 1818) et Linton signale bien la présence d'embryons fusiformes dans la cavité générale de l'unique exemplaire sur lequel il a basé sa description. En fait, cette femelle a été trouvée par hasard dans le flacon qui contenait des larves d Acanthocéphales enkystées dans la paroi digestive ou le mésentère du requin 
Provenait-elle bien de l'intestin du squale ou bien d'un autre hòte ? Les conditions de cette récolte sont trop suspectes pour que nous puissions alfirmer quoique ce soit à son propos et nous avons jugé plus prudent de ne pas en tenir compte dans notre travail. Dans la littérature à laquelle nous avons pu avoir accès nous n'avons pas trouvé d'autre exemple de Sélacien hébergeant des femelles ovigères. Pourquoi n'y a-t-il pas d'Acanthocéphales adultes chez les Chondrichthyiens ? Deux hypothèses peuvent être avancées sans qu'il soit possible d'aller au-delà :

— ou bien, lorsqu'à partir des formes ancestrales libres, les Acanthocéphales se sont adaptés à la vie parasitaire, les Cyclostomes et les Sélaciens étaient déjà des lignées anciennes et l'option pour le parasitisme des Acanthocéphales primitifs a coïncidé avec l'apparition des Ostéichthyiens au milieu du Dévonien ;

- ou bien, le milieu intestinal de ces Vertébrés, bien différent de celui des Poissons osseux, ne permet pas l'implantation puis le développement jusqu'au stade adulte des juvéniles d'Acanthocéphales. Cette seconde hypothèse devrait pouvoir ètre vérifiée expérimentalement. Elle expliquerait aussi pourquoi les Acanthocéphales n'ont pas réussi à être des "parasites de capture " chez les Cyclostomes et les Sélaciens.

En fait, ces deux hypothèses peuvent être complémentaires, l'ụn n'excluant pas l'autre.

Chez les Acipensériformes, seul le genre Leplorhynchoides a été signalé et nous l'avons considéré, de par ses caractères morphologiques, comme primitif parmi les Palaeacanthocephala.

Les Albulidae, Clupéiformes primitifs, sont parasités par des Illiosentidae (Illiosentis et Tegorhynchus), Famille que nous considérons comme la plus "primitive " des Palaeacanthocephala.

Une autre Famille de Clupéiformes "primitifs ", celle des Osteoglossidae, héberge d'une part des Tenuisentidae, les moins évolués des Eoacanthocephala et des Polyacanthorhynchidae, Famille incertae sedis, possédant des caractères qui la rapproche à la fois des Palaeacanthocephala et des Eoacanthocephala.

\section{2 - Du point de vue des Acanthocéphales}

Mis à part les quelques exemples que nous venons de donner, il nous paraît impossible d'établir un parallèle entre la phylogénie des poissons hôtes définitifs et celle des Acanthocéphales qu'ils hébergent. Il semble évident que beaucoup doivent être des " parasites de capture " qui, grâce à leur plasticité adaptative, ont réussi à s'établir chez de nouveaux hôtes et à y boucler leur cycle.

Si l'on considère séparément les poissons marins et les poissons d'eau douce, on constate que certains genres se rencontrent indifféremment chez les uns et chez les autres (exemple : Pomphorhynchus, Acanlhocephalus, Echinorhynchus, Neoechinorhynchus). En fait, il s'agit de poissons marins littoraux dont on trouve des espèces congénériques ou très voisines installées définitivement en eau douce. Un tel résultat pouvait facilement être prévu. 
Quant aux poissons de grande profondeur, ils hébergent des Echinorhynchus ou des proches de ce genre.

Il serait certainement intéressant d'étudier également la faune des Acanthocéphales en fonction du comportement trophique des Poissons hôtes, en particulier de distinguer ceux qui se nourrissent en surface de ceux qui se nourrissent au fond. On sait aussi que le nombre de poissons changent d'alimentation en fonction de leur âge. Signalons encore que les Scombridae et les Thunnidae, prédateurs de haute mer, hébergent presque exclusivement des Rhadinorhynchidae alors que les Mugilidae, microphages littoraux, sont parasités seulement par des Neoechinorhynchidae.

Enfin, alors que certains Acanthocéphales apparaissent strictement inféodés à un groupe restreint d'hôtes, voire à un seul hôte $(2,3,36)$, d'autres sont très largement distribués. C'est le cas d'Acanthocephalus dirus d'Amérique du Nord qui s'oppose ainsi à Acanthocephalus tahlequahensis et à $A$. alabamensis, également nord-américains dont l'aire de répartition et le nombre d'hôtes définitifs "vrais" sont très limités. L'exemple le plus remarquable est celui de Serrasentis sagittifer qui n'a été trouvé parfaitement mûr que chez les Rachycentridae et les Carangidae, deux Familles de Perciformes extrêmement voisines. L'hôte type, Rachycentron canadum, est un poisson pélagique très largement distribué dans les régions intertropicales de l'Atlantique, de l'Océan indien et du Pacifique Ouest et il est parasité par cette espèce d'Acanthocéphale depuis les côtes atlantiques des U. S. A. à celles du Sénégal et de l'Inde. Chez tous les autres poissons chez lesquels $S$. sagittifer a été signalé, et la liste en est très longue, ce parasite est toujours sous forme de larve enkystée dans la cavité générale ou, à la rigueur, de juvénile implanté dans l'intestin. Tous ces poissons sont des hôtes d'attente indispensables à la réalisation du cycle du parasite, assurant le passage du Crustacé hôte intermédiaire au prédateur ichtyophage, hôte définitif.

\section{Conclusion}

Au terme de cette étude qui doit être considérée comme tout à fait préliminaire, il nous apparaît que la spécificité des Acanthocéphales adultes est bien plus grande qu'il n'était classique de le dire. Certes, la liste des poissons-hôtes est très longue mais elle se réduit considérablement lorsque l'on introduit la notion de présence ou d'absence de femelles ovigères.

Quelques genres d'Acanthocéphales que nous avons considérés comme "primitifs " en nous basant sur des caractères purement morphologiques apparaissent bien inféodés à des poissons également " primitifs ". Mais, dans l'immense majorité des cas, le facteur déterminant du parasitisme est sans aucun doute le comportement trophique du poisson.

Si quelques espèces sont strictement liées à un groupe d'hôtes définitifs spécifiques, voire à un hôte précis, la plupart parviennent au stade de complète maturité 
sexuelle des femelles chez des poissons appartenant à des Familles très diverses, ce qui confirme la plasticité adaptative des Acanthocéphales. Il faut cependant nuancer cette affirmation car il y a des exemples précis d'espèces dont les femelles ovigères ont été effectivement trouvées chez des hôtes que l'on doit considérer sinon comme des hôtes accidentels, mais tout au moins, comme des hôtes d'exception, rarement contaminés ou n'hébergeant que quelques rares femelles mûres. Ces hôtes ne doivent avoir qu'un rôle très marginal dans la réalisation habituelle du cycle du parasite dans la nature.

Il faut aussi tenir compte de la saison, certains hôtes n'hébergeant une espèce d'Acanthocéphale parfaitement mûre qu'à certaines périodes très déterminées de l'année.

Nous jensons que dans les travaux à venir, il serait nécessaire que les auteurs précisent toujours le degré de maturité sexuelle des femelles et indiquent avec précision le nom de l'hôte chez lequel elles ont été trouvées. Nous obtiendrons ainsi une meilleure appréciation de la spécificité des Acanthocéphales pour leurs hôtes définitifs. Il se peut qu'en ce domaine nous ayons quelques surprises !

Remerciements. - Nous tenons à exprimer nos remerciements à Francine Breton qui, avec patience et gentillesse, a accepté de passer des heures devant l'écran de l'ordinateur pour composer le tableau des Acanthocéphales et de leurs Poissons-hôtes.

\section{BIBLIOGRAPHIE}

1. Acimenov A. K. : Acanthocéphales des poissons du fleuve Amour (en russe). Trud. Gelminthol. Laborat., 1959, 9, 23-44.

2. A Min O. M. : Variability and redescription of Acanthocephalus dirus (Acanthocephala, Echinorhynchidae) from freshwater fishes in North America. Proceed. Helminth. Soc. Washington, $1984,51,225-237$.

3. Amrv O. M. : Hosts and geographic distribution of Acanthocephalus (Acanthocephala, Echinorhynchidae) of North American freshwater fishes, with a discussion of species relationships. Proceed. Helminthol. Soc. Washington, 1985, 52, 210-220.

4. Amin O. M., Nahilas F. M., Az-Yamani F., Abu-Hakina R. : On three acanthocephalan species from some Arabian Gulf fishes off the coast of Kuwait. $J$. Parasitol., 1984, 70, 168-170.

5. AMrN O. M., REDLin M. J. : The effect of the host species on growth and variability of Echinorhynchus salmonis Muller, 1784 (Acanthocephala-Fchinorhynchidae), with special reference to the status of the genus. Systematic. Parasitol., 1980, 2, 9-20.

6. Avantaraman S. : Acanthosentis oligospinus n. sp., an acanthocephalan parasite of the brackish water fish, Mystus (Macrones) gulio, in Madras. Indian J. Parasitol., 1980, 4, 21-24.

7. Andrews Ch., Rojaxapaibul A. : The ecology of Acanthocephalus clavila (Dujardin, 1845) in the fish of I.lyn Tegid, North Wales. Parasitol., 1976, 73, 11.

8. BAYlis H. A. : Some parasitic worms from Arapaima gigas (Teleostean fish) with a description of Philometra senticosa n. sp. (Filaroidea). Parasitol., 1927, 19, 35-47.

9. BAYLIS H. A. : Parasitic Nematorla and Acanthocephala collected in 1925-1927. Discovery Rep., 1929, 1, 541-560.

10. Bravo Hollis M. : Helmintos de peces del Pacifico Mexicano. 28. Sobre dos especies del género Floridosentis Ward, 1953, Acantocéfalos de la Familia Neoechinorhynchidae Van Cleave, 1919. Ann. Instit. Biol. Univ. Nacion. Auton. Mexico, 1969, 40 (sér. Zool.), 1-14.

11. BUCKNER R. I. : Occurrence of two species of Neoechinorhynchus (Acanthocephala) in the golden shiners of Alabama and Mississipi. Proceed. Ilelminth. Soc. Washington, 1983, zo, $176-178$.

12. Bullock W. I.. A redescription of Octospiniferoides chandleri Bullock, 1957. J. Parasitol., $1966,52,735-738$. 
13. Bunon I. DE : Biologie des populations d'Acanthocéphales. F́tude du complexe Acanthocephaloides propinquus, parasite de Poissons marins et lagunaires. Thèse Sci., Montpellier, $1986,210 \mathrm{p}$.

14. Buron 1. DE, Golvan Y. J. : Les hôtes des Acanthocéphales. I. Les hòtes intermédiaires. Ann. Parasitol. Hum. Comp., 1986, 61, 581-592.

15. Снивв J. C. : Occurrence of Echinorhynchus clavala (Dujardin, 1845) nec Hamann, 1892 (Acanthocephala) in the fish of Llyn Tegid (Bala Lake), Merionethshire. J. Parasilol., 1964, $50,52-59$.

16. Degrusti D. L. : The life cycle of Leplorhynchoides thecatus (Linton) an Acanthocephalan of fish. J. Parasitol., 1949, 35, 437-460.

17. Dollfus R. Ph. : De Raorhynchus terebra (Rudolphi) (Palaeacant hocephala, Rhadinorliynchidae). Bull. Mus. Nat. Hisl. Nalur., 1969, 41 (2e sér., 3), 755-758.

18. Enmosos S. J. : Acanthocephala collected by the Australian National Research Expedition on Heard Island and Macquarie Island during 1948-1950. Transac. Roy. Soc. South Australia, $1955,78,141-144$.

19. Edmonds S. J. : Australian Acanthocephala no 10. Transac. Roy. Soc. South Austrulia, 1957, 80, 76-80.

20. Edmonds S. J. : Australian Acanthocephala no 15 ; four species. Transac. Roy. Soc. Sonth Australia, 1982, 106, 71-76

21. Faroodi H. U.: Acanthocephala from marine fishes of Nigeria. Indian J. Parasitol., 1981, $5,125-131$.

22. Fernando C. H., Funtado J. I. : A study of some helminth parasites of freshwater fishes in Ceylon. Zeitschr. Parasilenkd., 1963, 23, 141-163.

23. FukUi T., Morisita T. : Notes on the acanthocephalan fauna of Japan. Annot. Zool. Japonen, $1938,17,567-576$.

24. George P. V., Nadakal A. M. : Studies on the life cycle of Pallisentis nagpurensis Bhalerao, 1931 (Pallisentidae : Acanthocephala) parasitic in the fish Ophiocephalus striatus (Bloch). Hydrobiol., 1973, 42, 31-43.

25. Golvan Y. J. : Une espèce et une variété nouvelles d'Acanthocéphales parasites des poissons de mer des côtes du Sénégal et redescription de Serrasentis socialis (Leidy, 1851) Van Cleave, 1924. Ann. Parasilol. Hum. Comp., 1956, 31, 225-241.

26. Golvan Y. J., Houin R., Deltoun H.: Nipporhynchus africanus n. sp. (Palaeacanthocephala) parasite d'un Carangidae du Gabon. Bull. Soc. Zool. France, 1963, 88, 71-85.

27. Golvan Y. J., Mokhayer B. : Acanthocéphales des esturgeons de la mer Caspienne. Ann. Parasitol. Hum. Comp., 1973, 48, 597-602.

28. Grabda-Zazubska B., E.JSymont L. : Studies on morphology variability and systematic status of Echinorhynchus borealis Linstow, 1901 (Acanthocephala, Echinorhynchidae). Acta Parasitol. Polon., 1969, 17, 65-87.

29. Gupta N. K., JaIN M. : On three already known species of the genus Neoechinorhynchus Itamann, 1892 (Acanthocephala) from fish of the Arabian sea. Acta Parasit. Polon., 1983, $18,407-416$.

30. Gulpa N. K., I.ata V. : On six new species of Acanthocephala from some vertebrate hosts in India. Res. Bull. (N. S.) Panjab Univers., 1967, 18, 253-268.

31. Gupta N. K., LATA V. : Observations on eight already known Acanthocephalan parasites from vertebrate hosts. Res. Bull. (N. S.) Panjab Univers., 1967, 18, 325-341.

32. Haley A. J., Bullock W. L. : A new species of Acanthocephala from the sunfish Lepomis gibbosus (I.inné) with a redescription of the Family Fessisentidae Van Cleave, 1932. Amer. Midland Nalural., 1953, 50, 202-205.

33. Harada I. : Zur Acanthocephalen fauna von Japan. Mem. Fac. Sci. Agric. Taihoku Imper. Univ. Formosa, Japan, 1935, 14, 7-23.

34. Harada I. : Acanthocephalen aus Formosa (I). Annot. Zool. Japon., 1938, 17, 419-427.

35. HAнтwich G. : Südamerikanische Acanthocephalen aus der Zoologischen Sammlung (les I3ayerische Staates. Zoolog. Anzeig., 1956, 156, 299-308.

36. Hubschmax J. H. : Diaptomus pallidus Herrick, 1879 (Crustacea, Copepoda) as an intermediate host for Tanaorhamphus longirostris (Van Cleave, 1913) (Acanthocephala, Neoechinorhynchidae). J. Parasitol., 1983, 69, 930-932.

37. Icilihara A., Kato K., Kamegai S., Machida M. : On the parasites of fishes and shellfishes in Sagami Bay. (no 4) Parasitic Helminths of the mackrel, Pneumalophorus japonicus (Houttuyn). Res. Bull. Meguro Parasit. Mus., 1968, 2, 45-57.

38. Jain M., Gupta N. IK. : On two already known species of the genus Pallisenlis Van Cleave, 1928 and discussion on the validity of Pallisentis buckleyi Tadros, 1966 and genus Devendrosentis Sahay, Sinha et Ghosh, 1971. Helminthol., 1979, 16, 173-183.

39. JAIN M., GUPTA N. K. : On some already known species of the genus Centrorhynchus Lühe, 1911 (Acanthocephala) from varjed vertebrate hosts in India. Res. Bull. Panjab Ininers., $1983,34,15-17$.

40. JILEK R.: Seasonal occurrence and host specificity of Gracilisentis gracilisenlis and Tunaorhamphus longirostris (Acanthocephala, Neoechinorhynchidac) in Crab Orchard lake, Illinois. J. Parasitol., 1978, 64, 951-952. 
41. Johnston T. H., Evmonbs S. J. : Australian Acanthocephala no 6. Rec. South Australian Mus., 1947, 8, 555-562.

42. Johxston T. H., Edmonds S. J. : Australian Acanthocephala no 9. Transac. Rou. Soc. South Australia, 1952, $75,16-21$.

43. Kostylew N. N. : Le gente Leptorhynchoides, nouveau genre d'Acanthocéphale parasile de Poissons. Ann. Parasitol. Hum. Comp., 1924, 2, 214-223.

44. Kostylew N. N. : 1. Zur Kenntnis der Acanthocephalen der Fisches des Schwarzen Meeres. Zoolog. Anzeig., 1926, 67, 177-183.

45. LEe R. I.. G. : Ecology of Acanthocephalus lucii (Müller, 1776) in perch, Perca fluviatilis, in the Serpentine, London, U. K. J. Helminthol., 1981, 55, 149-154.

46. Leong T. S., Holmes J. C. : Acquisition of helminths by coho salmon, Oncorhynchus kisutch introduced into Cold Lake, Alberta: a comparison with helminths of native salmonid fishes. IIId Internat. Congr. Parasitol. München, 1975, 3, edit. Vienna (Austria).

47. I.xcicome D. R., Van Cleave H. J. : Distribution of Leptorhynchoides thecatus, a common acanthocephalan parasite in fishes. Am. Midland Natural., 1949, 41, 421-431.

48. Luxuström A. : Die Acant hocephalen Schwedens mil ausnahme der Fischacanthocephalen von Süsswasserstandorten. These Sci. Lund (Suede), 1942, 238 p.

49. I,YNCH J. E. : New species of Neoechinorhynchus from the Western Sucker, Catostomus macrocheilus Girard. Transac. Am. Microsc. Soc., 1936, 55, 21-43.

50. Machado Filio D. A. : Revisao do gênero "Polyacanlhorhynchus "Travassos, 1920 (Acanthocephala, Rhadinorhynchilae). Rev. Brasil. Biol., 1947, 7, 195-201.

51. MAchado Filio D. A. : Uma nova espécie do gênero "Neoechinorhynchus "Hamann, 1892 parasita de "Peixe-Martin " do Paraguai (Neoechinorhynchidae, Archiacanthocephala). liev. Brasil. Biol., 1959, 19, 379-381.

52. Machina M., ARAKi J. : Redescription of Pseudorhadinorhynchus leuciscus (Krotov et Petrotschenko, 1956). Res. Bull. Meguro Parasit. Mus., 1982, 8, 49-51.

53. Maxilla G., Orecchia P., Paggi L. : Parassitofauna di Salmo trulla l. del Fiume Tirino. Nota I; redescrizione di Dentilruncus iruttae Sinzar, 1955 et considerazioni sul genere Dentitruncus Sinzar, 1955. Parassilol., 1976, 18, 71-78.

54. Marchanis B. : A comparative ultrastructural study of the shell surrounding the mature acanthor larvae of 13 Acanthocephala species. I. Parasitol., 1984, 70, 886-901.

55. Marchand B., Mattei X. : I,a spermatogenèse des Acanthocéphales. I. I'appareil centriolaire et flagellaire au cours de la spermiogenèse d'Illiosentis furcalus var. africana Golvan, 1956 (Palaeacanthocephala, Rhadinorhynchidae). J. Ultrastruct. Res., 1976, 54, 347-358.

56. Masi-Pallares R. C., Bexitez-L'sher A., Vergara G. : Rev. Paraguay Wicrobiol., 1973,8 , 67-112 [cité par Nickol et PabILha sans titre (1979)].

57. Meade T. G., Harvey J. S., Jr. : New records of numbers and sites of infection in fishes by the Acanthocephalan Eocollis arcanus Van Cleave. J. Parasitol., 1968, 54, 371.

58. Merritt S. V., Pratt I. : The life history of Neoechinorhynchus rutili and its development in the intermediate host (Acanthocephala, Neoechinorhynchidae). J. Parasitol., 1964, 50, $394-400$.

59. Meyer-Thill C., Hoffmann J. : Contribution à l'étude de l'Acanthocéphale Echinorhynchus trultue (Schrank), parasite des Truites (phase liée à l'hôte principal). Arch. Instit. Gid. Duc. luxembourg, 1970, 34, 251-296.

60. Nickol B. B., Padilha T. N.: Neoechinorhynchus paraguayensis (Acanthocephala, Neoechinorhynchidae) from Brazil. .J. Parasitol., 1979, 65, 987-989.

61. Nomoxin I). : Remarks on Neoechinorhynchus caremai Noronha, 1973 (loacanthocephala, Neoechinorhynchidae). Mem. Instil. Oswaldo Cruz., 1984, 79, 271.

62. Oetraer D. F., Buckser R. I. : Acanthocephalus tahlequahensis sp. n. (Acanthocephala, Echinorhynchidae) from the stippled Darter, Etheostoma punclulatum (Agassiz), in Northern Oklahoma. J. Parasitol., 1976, 62, 237-241.

63. OLsox IR. 1.., P'RATt I. : The life cycle and larval development of Echinorhynchus lageniformis Ekbaum, 1938 (Acant hocephala, Echinorhynchidae). J. I'arasilol., 1971, 57, 143-149.

64. RaI I'. : On four acanthocephalan genera parasitic in fresh water fishes with description of three new species. Indian J. Helminthol., 1967, 19, 27-44.

65. Salgado-MaLdonano G. : Acantocefalos de Peces. III. Redescripcion de Dollusentis chandleri Golvan, 1969 (Acanthocephala, llliosentidae) y descripcion de una nueva especie del mismo genero. Ann. Instit. Biol. Univ. Nac. Auton. Mexico, 1976, 47 (2, ser. Zool.), 19-34.

66. Salgado-Maldonado G. : Acantocéfalos de Peces. V. Redescripcion de cuatro especies de Palaeacantocefalos parasitos de peces de Mexjco. Ann. Instit. Biol. Univ. Nac. Auton. Mexico, 1978,49 (1, ser. Zool.), 49-70.

67. Salgado-Maldoxado G. : Acantocéfalos de Peces. VI. Hallazgo de Gorgorhynchoides bullocki Cable y Mafarachisi, 1970 (Acanthocephala : Arhythmacanthidae) y descripcion de algunos de sus estadios juveniles. Ann. Instit. Biol. Univ. Nac. Aulon. Mexico, 1979, 50 (1, ser Zool.), $35-50$.

68. Salgado-Maldonado G. : Crecimiento alometrico y consideraciones laxonomicas sobre Neoechinorhynchus golvani Salgado-Maldonado, 1978 (Acanthoccphala : Neoechinorhynchidae) parasilo de peces dulceacuicolas en Tabasco, Mexico. Univers. I Cienc., 1985, 2, $57-66$. 
69. Salgado-Maldonado G., Barquin-Alvarez N. P. : Floridosentis elongatus Ward, 1953 y Contracaecum sp. parasitos de Mugil cephalus Linnaeus, 1758. Ann. Instit. Biol. Univ. Nac. Auton. Mexico, 1978, 49 (1, ser. Zool)., 71-82.

70. Samuel G., Bullock W. L. : Life cycle of Paratenuisentis ambiguus (Van Cleave, 1921) Bullock et Samuel, 1975 (Acanthocephala, Tenuisentidae). J. Parasitol., 1981, 67, 214-217.

71. Samuel N., Nickol B. B., Mayes M. A. : Acanthocephala of Nebraska fishes. Am. Midld. Natural., 1976, 96,391-406.

72. Sandeman I. M., Prppy J. H. C.: Parasites of freshwater fishes (Salmonidae and Coregonidae) of insular Newfoundland. J. Fish. Res. Board. Canada, 1967, 24, 1922-1927.

73. Sснмгот G. I. : A note on the acanthocephala parasitizing amphipod crustacea in a springfed pond in Montana. Can. J. Zool., 1964, 42, 718 .

74. Schmid G. D. : A collection of Acanthocephala from fishes of George Lake, Central Alaska. Can. J. Zool., 1965, 43, 651.

75. Scirmint G. D., Huggins E. J. : Acanthocephala of South American Fishes. Part 2. Palaeacanthocephala. J. P'arasitol., 1973,59, 836-838.

76. Stranack F. R. : The fine structure of the acanthor shell of Pomphorhynchus laevis (Acanthocephala). Parasitol., 1972, 64, 187-190.

77. Troncy P. M., Vassiliades G. : Acanthocéphales parasites des Poissons d'Afrique. Bull. Instil. Fond. A/rique Noire, 1973, 35, 522-539.

78. UGLEM G. I.., I.ARSON O. R. : The life history and larval development of Neoechinorhynchus saginatus Van Cleave et Bangham, 1949 (Acanthocephala, Neoechinorhynchidae). J. Parasitol., $1969,55,1212-1217$.

79. UzNaNski R. L., Nickol B. B. : A sequential ranking system for developmental stages of an acanthocephalan, Leptorhynchoides thecatus, in its intermediate host, Hyalella azteca. .J. Parasitol., 1980, 66, 506-512.

80. Van Cleave H. J. : Acanthocephala from the Subfamily Rhadinorhynchidae from American fishes. J. Parasitol., 1918, 5, 17-27.

81. Van Cleave H. J. : Acanthocephala from the Illinois River, with descriptions of species and a synopsis of the family Neoechinorhynchidae. Bull. Illinois Stat. Nat. Hist. Surv., 1919, $13,225-257$.

82. Van Cleave H. J. : Acanthocephala of fishes of Douglas Lake, Michigan. Occas. Pap. Mus. Zoolog. Univers. Michigan, 1919, n० 72, 1-12.

83. Van ClEave H. J. : Two new genera and species of acanthocephalous worms from venezuelan fishes. Proceed. U.S. Nat. Mus., 1920, 58, 455-466.

8.1. Van Cleave H. J. : Acanthocephala from the fishes of Oneicla Lake, New York. Roosevelt Wild Life Bull., 1923, 2, 73-84.

85. VAN (CLEAvE H. J., IYYNCh J. E. : The circumpolar distribution of Neoechinorhynchus rutili, an acanthocephalan parasite of fresh-water Fishes. Transac. Am. Microsc. Soc., 1950, 59, $156-171$.

86. VAN MAREN M. J. : The Amphipod Gammarus fossarum Koch (Crustacea) as intermediate host for some helminth parasites, with notes on their occurrence in the final host. Bii. Tot Dierkd., 1979, \&8,97-110.

87. Walkey M. : The ecology of Neoechinorhynchus rutili (Müller). J. Parasitol., 1967, 53, 795-804

88. Wakb H. 1. : Studies in the life history of Neoechinorhynchus cylindratus (Van Cleave, 1913) (Acanthocephala). Transac. Am. Micros. Soc., 1940, 59, 327-347.

89. Williams J. S., Gibson D. I., Sadighian A. : Some helminth parasites of Iranian fresh water fishes. $J$. Nat. Hist., $1980,14,685-699$.

90. Yamaguti S. : Studies on the Helminth Fauna of Japan. Part 8. Acanthocephala, I. Jpn. J. Zool., 1935, 6, 247-278.

91. Zozitowiecki K. : Antarctic Acanthocephalans of the genus Metacanthocephalus., Acta. Parasil. Pol., 1983, 28, 417-437.

92. Zozitowiccki K. : Acanthocephala of the Antaretic. Pol. Polar Res., 1986, 7, 79-117.

93. ZDzitowiecki K. : Acanthocephala of marine fishes in the regions of South Georgia and South Orkneys (Antarctic). Acta Parasitol. Polonica, 1987, 31, 211-217.

9.1. ZscrokKe F. : Recherches sur l'organisation et la distribution zoologique des vers parasites des Poissons d'eau douce. Dissert. Fac. Sci. Genève, 1884, 89 p. 